\title{
Status of Intraductal Therapy for Ductal Carcinoma in Situ
}

\author{
Meghan Flanagan • Susan Love • E. Shelley Hwang
}

Published online: 6 May 2010

(C) The Author(s) 2010. This article is published with open access at Springerlink.com

\begin{abstract}
The intraductal approach is particularly appealing in the setting of ductal carcinoma in situ (DCIS), a preinvasive breast neoplasm that is thought to be entirely intraductal in its extent. Based on an emerging understanding of the anatomy of the ductal system as well as novel techniques to leverage the access accorded by the intraductal approach, researchers are actively exploring how ductal lavage, ductoscopy, and intraductal infusion of therapeutic agents may enhance breast cancer treatment. Both cytologic and molecular diagnostics continue to improve, and work is ongoing to identify the most effective diagnostic biomarkers for DCIS and cancer, although optimal targeting of the diseased duct remains an important consideration. Ductoscopy holds potential in detection of occult intraductal lesions, and ductoscopically guided lumpectomy could increase the likelihood of a more comprehensive surgical excision. Exciting pilot studies are in progress to determine the safety and feasibility of intraductal chemotherapy infusion. These studies are an important starting point for
\end{abstract}

\section{Flanagan}

Albert Einstein College of Medicine,

New York, NY, USA

S. Love

David Geffen School of Medicine

at University of California, Los Angeles,

Los Angeles, CA, USA

E. S. Hwang $(\bowtie)$

Division of Breast Surgery, San Francisco Department of Surgery, University of California,

San Francisco, CA, USA

e-mail: Shelley.Hwang@ucsfmedctr.org

E. S. Hwang

Helen Diller Comprehensive Cancer Center,

San Francisco, CA, USA future investigations of intraductal ablative therapy for DCIS, because as our knowledge and techniques evolve, it is likely that DCIS may be the target most amenable to treatment by intraductal therapy. If such studies are successful, these approaches will allow an important and meaningful transformation in treatment options for women diagnosed with DCIS.

Keywords Carcinoma in situ (DCIS) - Preinvasive cancer . Breast cancer $\cdot$ Intraductal therapy $\cdot$ Ductal lavage

\section{Introduction}

Ductal carcinoma in situ (DCIS) is the most common noninvasive lesion of the breast. It is defined as the proliferation of malignant ductal epithelial cells without penetration of the basement membrane. Greater use of screening mammography over the past few decades has led to increasing diagnosis of DCIS such that the incidence of DCIS increased from 1.87 per 100,000 women from 1973 to 1975 to 32.5 per 100,000 women in 2004 [1]. Approximately $25 \%$ of breast cancers currently diagnosed by mammography in the United States are classified as DCIS, and it has been estimated that more than 1 million women in the United States alone will be living with a diagnosis of DCIS by 2020 [1]. Despite the increasing prevalence and high mammographic detection rates, the natural history of DCIS continues to be poorly understood.

The linear model of breast carcinogenesis suggests that DCIS is an intermediate step between normal healthy breast tissue and invasive breast cancer [2]. Although a few retrospective studies evaluating misdiagnosed or biopsy only treatment of DCIS indicate that some $14 \%$ to $53 \%$ of patients will progress to invasive cancer without treatment, virtually no prospective data have been obtained regarding 
progression of untreated disease [3-6]. Substantial data indicate that recurrence rates for patients with DCIS are dramatically reduced after excision or mastectomy. Standard therapy for DCIS is surgical excision, with $31 \%$ of women treated with mastectomy, $28 \%$ with breast-conserving surgery alone, and $40 \%$ with breast-conservation therapy and radiotherapy [7]. In a study by Silverstein et al. [8] comparing local recurrence among patients with DCIS, 7 -year disease-free survival rates were approximately $98 \%$ in those choosing mastectomy versus $84 \%$ in those receiving breast-conservation surgery with radiation. However, this same study, as well as others, has failed to show a survival benefit from any treatment modality used to treat DCIS [8].

Because patient perception of the risk of developing local recurrence or dying of breast cancer does not differ significantly whether diagnosed with DCIS or invasive breast cancer $[9,10]$, it can be inferred that patients will continue to pursue aggressive therapies that could pose considerable emotional and physical impact. Recent advances in technology and the development of new treatment modalities for DCIS have exploited the intraductal nonsystemic nature of DCIS by focusing on treatments specifically targeted to intraductal delivery. It is hoped that as these approaches are developed, patients will be provided less invasive treatment options for this disease. The intraductal approach includes procedures and technologies such as nipple aspiration, ductal lavage, ductoscopy, and most recently, the introduction of antineoplastic treatment agents directly into the ductal-alveolar unit for both diagnosis and treatment of DCIS. This review focuses on the intraductal approach to the diagnosis and treatment of breast cancer, with a specific emphasis on its role in the management of DCIS.

\section{Breast Ductal Anatomy}

The study of breast duct anatomy dates back to 1840 , with Cooper's studies injecting colored wax into the ducts of over 200 breasts of women who died during lactation [11]. He described human breast tissue as organized into separate lobes consisting of one central duct, its peripheral branches, and associated glandular tissue [11]. Subsequent to this report, a considerable number of studies using histologic, ductal, and imaging approaches have been conducted in an effort to describe the number and arrangement of ducts composing the human mammary gland [12].

Reports of breast and nipple duct anatomy vary depending on whether the study focused on openings on the nipple that can be cannulated in vivo or in vitro and followed into functional ducts in the breast or the number of duct profiles seen on cross-sections of the nipple. Original histologic studies and anatomy texts describe breast anatomy as radially symmetrical lobes with 15 to 20 central ducts. Ductogram studies by Sartorius and Smith demonstrated 5 to 9 ducts per breast, whereas Teboul and Halliwell described 15 to 20 ducts converging on 5 to 8 milk pores in a series of more than 6,000 breasts studied via ultrasound and ductoscopy [13]. Moffat and Going's [14] threedimensional (3D) computer model of a single autopsy breast traced 10 complete nonanastamosing ductal systems. Using six separate approaches, Love and Barsky [15] found that more than $90 \%$ of the nipples examined in their studies contained 5 to 9 ductal orifices. The distribution consisted of nonanastamosing central ducts that travel back toward the chest wall and peripheral ducts that drape over the central ducts in a radial fashion [15].

As a result of these and similar studies, it is now generally accepted that the ductal tree is a nonanastamosing system comprised of central ducts leading back to the chest wall and peripheral ducts draping over the central ducts. However, discrepancies remain regarding the number of ducts found on histologic cross sections and 3D image modeling versus the number of ducts visible and available for cannulation at the nipple surface. It still remains unclear whether these inconsistencies represent ductal branching close to the nipple surface or ducts with associated sebaceous glands, all of which have implications for intraductal approaches to management $[16,17]$.

\section{Intraductal Lavage}

The study of breast ductal fluid via nipple aspiration began in the 1950s, but the low yield of epithelial cells for cytologic analysis essentially precluded it as an efficient tool for diagnosis. In 2001, Dooley et al. [18] demonstrated significant success in producing cytologically evaluable fluid in ductal lavage specimens compared with nipple aspiration. The median epithelial yield from nipple aspiration was 120 cells per breast, whereas the yield with ductal lavage was 13,500 cells per duct [18]. Like nipple aspiration, ductal lavage is based on the principle that invasive breast cancer develops via a linear progression through intraductal precursor lesions, and that these lesions are located in the fluid-containing ducts. Accordingly, many have sought to discover whether ductal lavage may have a particular role in both diagnosis and treatment of intraductal cancers, which should be especially accessible via intraductal strategies.

A number of long-term follow-up studies have confirmed that the presence of ductal cellular atypia constitutes a risk factor for breast cancer. In a 17-year follow-up study, Page et al. [19] found that women with atypia on biopsy 
had 5.3 times the risk of developing invasive disease compared with women with nonproliferative lesions on biopsy. Wrensch et al. [20, 21] associated cellular atypia in nipple aspiration fluid with a 4.9 -fold higher relative risk than those women who produced no nipple fluid. Fabian et al. [22] used periareolar fine needle aspiration to assess women for the presence of atypia and found that $15 \%$ of women with an elevated Gail risk and atypia developed cancer compared with $4 \%$ of women with similar Gail risk but no atypia. Thus, the utility of ductal cytology in breast cancer risk stratification has been established, but the accuracy of ductal lavage for diagnosis of DCIS and invasive cancer remains a question. Investigators have evaluated the potential of both cytology and biomarker assessment of lavage fluid in this regard.

\section{Cytology of Intraductal Aspirates}

Because of the association of nipple aspirate fluid production and breast cancer risk, an assumption was made that fluid-yielding ducts were those most likely to harbor disease, and lavage of these ducts would yield cells with cytologic atypia corresponding to histologic abnormalities. However, multiple studies assessing the utility of cytologic evaluation have concluded that the ducts with precancerous or cancerous lesions are not necessarily more likely to yield fluid, and in addition, that the low sensitivity in detecting high-grade atypical cells makes cytologic evaluation of ductal lavage specimens a low-yield tool for assessing risk or identifying existing in situ or invasive breast cancer.

In women with known cancer undergoing mastectomy, Khan et al. [23] found that only $38 \%$ of cancer-containing ducts yielded ductal fluid for analysis, and Maddux et al. [24] determined that atypia rates were similar for both fluidproducing and dry ducts (19\% vs $15 \%)$. In Khan et al.'s study [23], only $36 \%$ of the fluid-containing ducts yielded ductal lavage specimens with marked atypia or malignancy, thus making the overall sensitivity for detecting cancer approximately $13 \%$. Brogi et al. [25] showed a similar sensitivity of $14 \%$ in their study of premastectomy ductal lavage on 26 cancer-containing breasts. Although a recent study by Wood et al. [26] found that nipple aspiration fluid was more commonly expressed in cancerous compared with unaffected breasts, overall sensitivity for detecting marked atypia or malignant cells in affected breasts was similar to previous studies, at approximately $17 \%$.

Sartorius et al. [27] had proposed that with increasingly large invasive cancers, the ducts might become obstructed and epithelial cells with higher-grade atypia may extravasate into surrounding tissues rather than shed into the ductal system. If this were the case, early noninvasive lesions such as DCIS would be more likely to yield atypical epithelial cells in lavage specimen. Interestingly, Chatterton et al. [28] did find that the presence of DCIS in the central ducts close to the nipple and the size of DCIS were independent predictors of atypical cytology, but most of the samples demonstrated only mild atypia on cytologic evaluation. In a follow-up study, Khan et al. [29] included only women with calcifications on mammogram, thus increasing the probability of DCIS lesions. Of the 10 women diagnosed histologically with DCIS, only one woman produced nipple fluid from a DCIS-bearing duct, and the cytology was benign [29]. These findings corroborate results of the earlier studies and suggest low overall utility for cytologic evaluation of ductal lavage, even in the setting of known DCIS.

\section{Molecular Biomarkers in Lavage Fluid}

Due to the relatively low yield of cytologic evaluation for breast cancer diagnosis, many groups have now focused on molecular biomarkers in ductal lavage fluid to identify characteristics specific to precancerous and cancerous lesions of the breast. Current studies analyzing genetic, epigenetic, and proteomic attributes of cells in ductal lavage fluid have suggested that biomarker analysis may be a more sensitive tool than cytology alone, because molecular biomarker changes often precede morphologic alterations and their detection might lead to earlier identification of cells at risk for malignant change.

Some investigators have used fluorescence in situ hybridization (FISH) to detect genomic abnormalities by identifying aneusomy of chromosomes within ductal lavage cells, as changes in copy numbers of chromosomes $1,8,11$, and 17 have been shown to be associated with both preinvasive and invasive breast lesions. King et al. [30] described aneusomy for these chromosomes in $71 \%$ of the specimens from malignant cases and in $11 \%$ from benign cases, for a sensitivity of $71 \%$ and specificity of $89 \%$. Krishnamurthy et al. [31] evaluated the utility of using FISH as an adjunct to cytologic evaluation. They confirmed chromosomal aneuploidy in all of the malignant and markedly atypical cases, but found aneusomy in only one case described by cytology as mildly atypical. The authors concluded that FISH-based detection of chromosomal aneuploidy could potentially be used as an adjunct to cytologic evaluation in confirming both benign and malignant diagnoses [31]. Adduci et al. [32] utilized array-based comparative genomic hybridization (CGH) in primary surgical specimens then tested matched ductal lavage specimens with region-specific FISH probes to determine whether similar chromosomal alterations were present in both specimens. Although only $11 \%$ of cytology samples were characterized as malignant, $55 \%$ of samples showed biomarker changes that were identical to those 
found in the primary surgical specimen. This study clearly demonstrated the increased sensitivity of molecular probes over cytology for detection of malignancy in ductal lavage samples [32].

Genomic and epigenomic markers have also been examined. Using quantitative fluorescence image analysis, Zhang et al. [33] evaluated lavage specimens from women with both benign and malignant findings and demonstrated $100 \%$ sensitivity in detecting cancer using DNA5cER and G-actin expression. Others have shown that evaluation of methylation may also have more sensitivity for cancer detection than cytology: Krassenstein et al. [34] used a panel of six loci to detect hypermethylation of $\mathrm{CpG}$ islands in matched tissue and nipple aspiration fluid samples. They detected hypermethylation in all of the malignant tissue and $82 \%$ of the fluid samples [34]. Fackler et al. [35] utilized quantitative multiplex methylation-specific polymerase chain reaction (QM-PCR) to evaluate promoter methylation events in a panel of genes believed to be associated with increased breast cancer risk. In their evaluation of QM-PCR versus cytologic evaluation, QM-PCR had double the rate of detection of cancer cells.

Proteomic analyses using the surface-enhanced laser desorption and ionization-time of light/mass spectrometry (SELDI-TOF-MS) technique have also been conducted on lavage fluid with success. Sauter et al. [36] prospectively collected nipple aspiration fluid from women scheduled for diagnostic breast surgery and identified three protein peaks associated with the pathologic categories of atypical ductal hyperplasia, DCIS, and invasive disease. With identification of additional proteins, this method may become a new tool for tumor detection.

At this time, the overall utility of nipple fluid as a tool for risk assessment and early detection of breast cancer remains controversial. Technical limitations of cannulation include duct perforation and lavage of non-cancercontaining ducts. Analysis of ductal fluid by cytology has been shown to have low sensitivity, with ambiguous predictive value associated with findings of mild atypia. However, the potential use of molecular biomarkers as a comprehensive panel or as an adjunct to cytologic analysis will continue to improve the diagnostic utility of ductal lavage as a minimally invasive method for risk assessment and early detection of breast cancer lesions.

\section{Ductoscopy}

Clearly, another way to exploit the access provided by the ductal system is through direct endoscopic visualization of the ductal tree, which may afford a novel approach to intraductal lesions such as DCIS. Although mammary ductoscopy (MD) has not yet garnered widespread use, tremendous improvements in endoscope technology in the past decade have allowed for unprecedented visualization and access to the intraluminal duct (Fig. 1). Both in the United States and Asia, MD has had the greatest utility in the setting of pathologic nipple discharge. Currently available endoscopes measure 0.55 to $0.9 \mathrm{~cm}$ in external diameter, often with a working side port that allows for snaring and extraction of ductal lesions. Investigators have shown that MD-guided extraction is effective for benign intraductal papillomas, but less effective in removing malignant lesions, including DCIS. Uchida et al. [37] have suggested two reasons for this observation, including a stiff and noncompliant ductal wall that is resistant to forceps biopsy, and the distal location of malignant lesions, many of which are located in the peripheral terminal ductal-lobular units.

In evaluation of abnormal nipple discharge, ductoscopy has been shown to be of benefit by avoiding the need for preoperative ductogram. One series reported that $61 \%$ of ducts with discharge had abnormal endoscopic findings that were surgically excised with ductoscopic guidance [38••]. Of the six cancers diagnosed, four had had negative preoperative radiographic work-up. In patients with known DCIS or breast cancer, ductoscopic guidance has been shown to improve reexcision rates. Dooley et al. [39••] have had extensive experience with this technique, and in their hands, lumpectomy for early-stage cancers guided by ductoscopy had a markedly reduced likelihood of local failure compared with standard lumpectomy techniques. As scope technology continues to improve, ductoscopy may
Fig. 1 Intraductal endoscopy. Current endoscopic technology has allowed high resolution visualization of intraductal anatomy and intraluminal abnormalities. a Normal duct, demonstrating smooth ductal walls and ductal bifurcation. b Exophytic lesion seen on ductoscopy of a duct with pathologic nipple discharge. Pathology of the excised lesion showed low-grade ductal carcinoma in situ (DCIS)
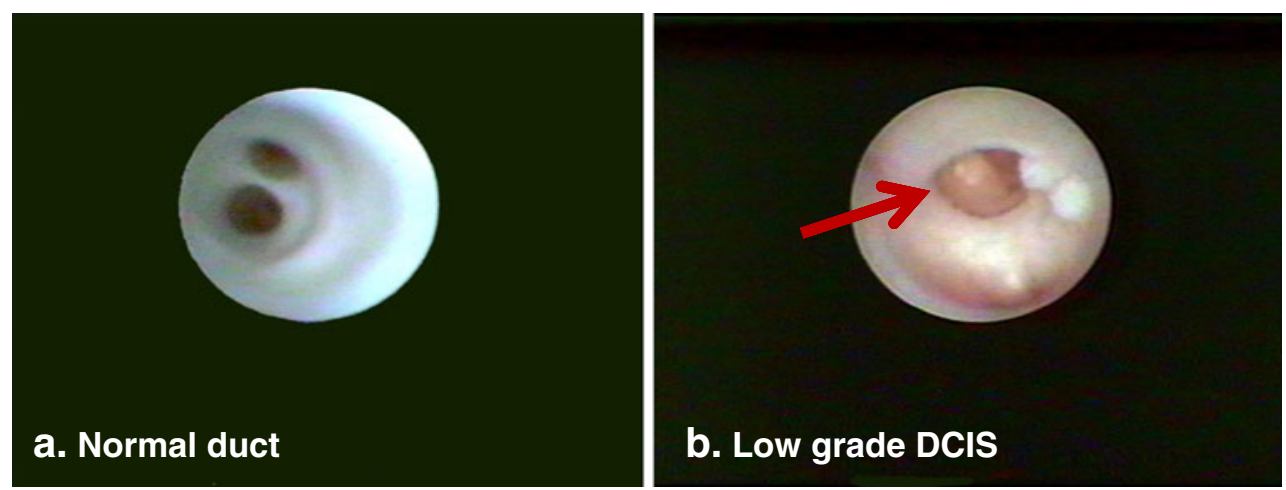
prove to be a useful localization option to guide resection of nonpalpable intraductal cancers.

\section{Intraductal Therapy}

Because most breast cancers arise from ductal epithelial cells, enormous opportunities exist in targeting therapy directly into the ductal system. Additionally, intraductal therapy is less likely to cause systemic toxicity and adverse effects. Currently, there are a number of phase 1 feasibility and safety trials evaluating the intraductal approach to breast cancer prevention and treatment.

Current clinical trials are based on intriguing initial animal studies by Murata et al. [40] that involved intraductal administration of the active tamoxifen metabolite 4hydroxytamoxifen (4-OHT) and pegylated liposomal doxorubicin (PLD) into transgenic mouse models of breast cancer. Results from the study demonstrated that not only was the intraductal administration of chemopreventive or chemotherapeutic drugs significantly more effective in preventing tumor development and promoting tumor regression, but there were also lower circulating levels of the agents and, as a result, no evidence of systemic toxicity. 4-OHT was found to be as effective as subcutaneous tamoxifen in the prevention of tumors, and intraductal administration of PLD caused complete regression in 24 of 25 tumors with a tumor-free 3-month follow-up period. PLD was also found to be protective against tumor formation. Toxicity studies showed no myelosuppression and peak levels of drug were significantly lower for intraductal versus intravenous injection.

As a result of the encouraging efficacy and safety data from these animal studies, a number of clinical trials are being conducted to explore the possibility of intraductal therapy in humans, primarily in the setting of DCIS or early-stage invasive cancer. Stearns et al. [41] at Johns Hopkins University School of Medicine have completed a phase 1 trial to determine the feasibility, safety, and maximum tolerated dose of PLD administered into one duct of women awaiting mastectomy. Preliminary data reveal that doxorubicin concentrations were not detectable in plasma, nipple aspirate fluid, or breast tissue [41]. Although histologic findings have not yet been reported, the safety of this intervention in women awaiting surgery for cancer appears promising.

In a similar Phase 1 study, Love and colleagues [42, 43] conducted a dose-escalation study in Beijing, China to determine safety and evaluate histopathologic response to either intraductal carboplatin or PLD instilled into 5 to 8 ducts in 32 women 2 to 7 days prior to mastectomy for breast cancer. Three dose levels were used, with the highest level approaching the dose used intravenously. At the highest doses of PLD, patients experienced tenderness and mild erythema and breast swelling, but no serious adverse events were noted. In the carboplatin group, both inflammatory responses and epithelial changes increased in a dose-dependent fashion. In the PLD group, no inflammatory changes were seen, but there was a marked increase of epithelial response to PLD treatment compared with the carboplatin-treated patients, including epithelial attenuation in the terminal ductal-lobular units (Fig. 2).

In a study directly targeted to test the practicality and efficacy of intraductal PLD treatment for DCIS, Love and Mahoney [44.•] are testing intraductal PLD in the neoadjuvant setting. In patients with core biopsy-proven DCIS, $20 \mathrm{mg}$ of PLD $\left(10 \mathrm{~cm}^{3}\right)$ is injected intraductally followed

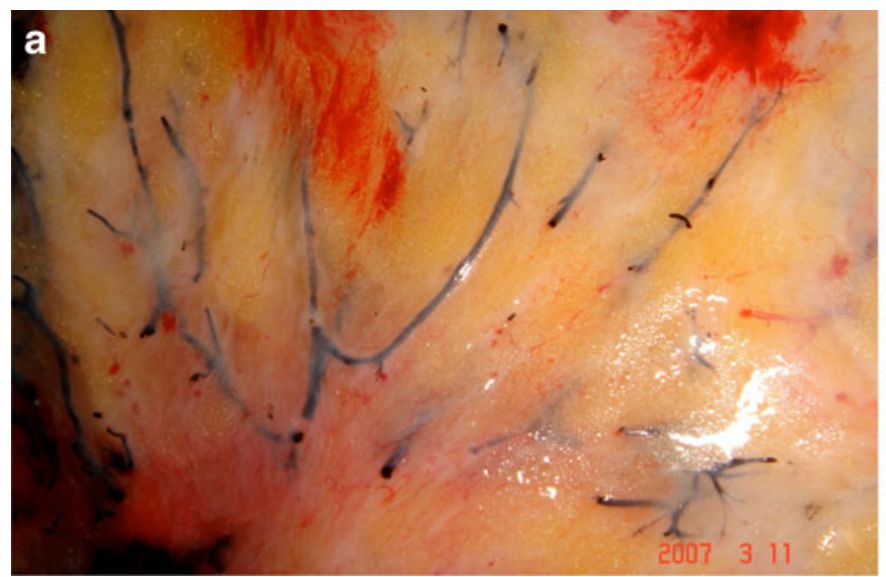

Fig. 2 Mastectomy specimen in a patient treated with intraductal pegylated liposomal doxorubicin (PLD). All patients in the study underwent surgery 2 to 5 days following PLD administration. Cannulated ducts were concurrently injected with dye to enable identification of treated ducts on pathologic evaluation. a Surgical

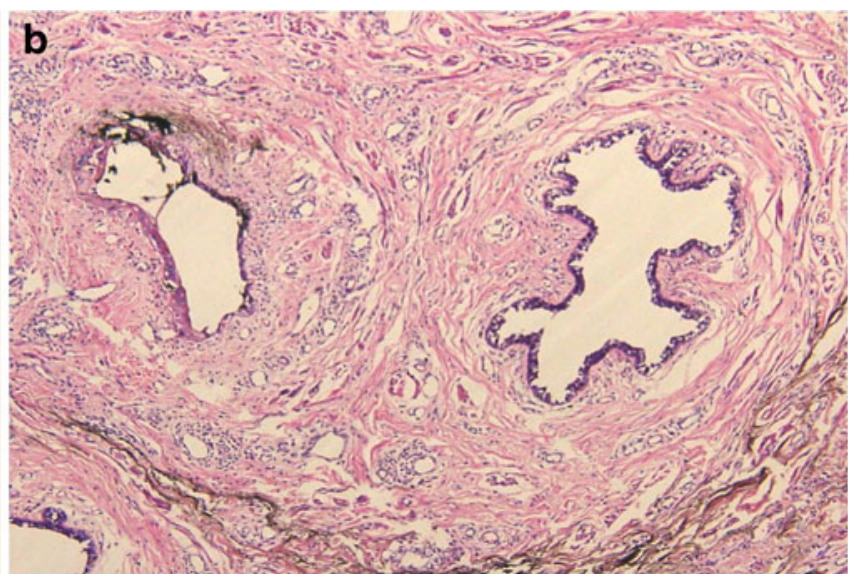

specimen with delineation of treated ducts by dye. The extent of ductal branching is clearly seen. b Hematoxylin and eosin (H\&E)stained section of treated duct (left) adjacent to untreated duct (right). The treated duct shows signs of marked epithelial atresia in response to PLD injection 
by a 6 -week observation period before scheduled surgery. The study end points are regression of the lesion on surgical pathology, decreased markers in ductal fluid, and changes on MRI before and after treatment. Thus far, four patients have received the full drug dose into the duct harboring DCIS, with two women reporting mild to moderate breast inflammatory reactions that responded to anti-inflammatory medications. Initial evaluation of histology of surgical specimens has confirmed a tissue response of inflammation, squamous metaplasia, and fat necrosis [44••]. This trial continues to accrue patients, and the investigators plan to collect data on tissue markers in the DCIS as well as in the cell pellet specimens collected at the time of PLD injection. Although women in this study will undergo surgery, the long-term implications of the trial may be to facilitate future treatment of DCIS with intraductal therapy alone. In addition, if this approach is effective in stripping cancer precursor cells from the ducts, intraductal injection of ablative agents in unaffected, high-risk women may allow for true loco-regional chemoprevention.

\section{Ductal Lavage for Monitoring DCIS}

Lavage biomarkers have been evaluated as potential indicators of response to systemic treatment, particularly in regard to analysis during repeat sampling in chemopreventive studies. Bhandare et al. [45] evaluated ductal lavage specimens from 145 high-risk women and found significant positive correlations between atypical cytology, epithelial cell number, and levels of the estrogen-related biomarkers Ki-67, estrogen receptor $\alpha$, and cyclooxygenase-2. As a follow-up study, these investigators conducted a phase 2 trial assessing the utility of ductal lavage to measure biomarkers of tamoxifen action [46••]. Of 182 women with Gail risk higher than $1.7 \%$ recruited to the study, $63 \%$ underwent entry and repeat ductal lavage and $47 \%$ had sufficient cells for analysis at both the initial and the follow-up lavage; 46 women chose observation and 39 women chose tamoxifen. The expected reduction in estrogen-related biomarkers was observed for $\mathrm{Ki}-67$ and estrogen receptor $\alpha$, but not in cyclooxygenase-2 in the tamoxifen-treated group. Additionally, cytologic findings showed a trend toward improvement in the tamoxifen group. Although the biomarker and cytologic profiles improved during the tamoxifen treatment, and thus seem to suggest clinical utility in this method of monitoring, the attrition rate and difficulty with obtaining adequate samples on repeat lavage make it necessary to re-evaluate this method [47]. However, as DCIS treatment evolves towards less invasive management strategies, women opting for active surveillance for DCIS may in the future be offered ductal lavage to monitor for cancer progression or response to systemic treatments.

\section{Conclusions}

The intraductal approach to the breast offers a unique opportunity to diagnose, study, and treat DCIS, a highly prevalent preinvasive lesion that is entirely confined within the ductal system. Additional studies are necessary to further elucidate the ductal system of the breast as well as the common anatomic distribution of DCIS within the system. The utility of intraductal lavage as a tool in the diagnosis and treatment of DCIS will hinge upon technologic advances that will allow for easier and more specific cannulation of affected ducts and continued research to establish a panel of biomarkers in addition to cytology that can reliably indicate disease. Thus far, small pilot trials have demonstrated that intraductal therapy is feasible and can be safely administered, and we await clinical trials of intraductal therapy alone to better evaluate the potential of this new and exciting therapeutic modality. The intraductal approach is of particular interest for disease confined to the ducts that does not require systemic therapy. It is hoped that continued advancements will offer women with breast cancer a minimally invasive way to detect breast disease, as well as in future allow for an important adjunct to surgical treatment for DCIS.

Disclosure No potential conflicts of interest relevant to this article were reported.

Open Access This article is distributed under the terms of the Creative Commons Attribution Noncommercial License which permits any noncommercial use, distribution, and reproduction in any medium, provided the original author(s) and source are credited.

\section{References}

Papers of particular interest, published recently, have been highlighted as:

•• Of major importance

1. Virnig BA, Shamliyan T, Tuttle TM, et al.: Diagnosis and Management of Ductal Carcinoma In Situ (DCIS): Evidence Report/Technology Assessment. Rockville, MD: Agency for Healthcare Research and Quality, U.S. Department of Health and Human Services; 2009:171

2. Lewis JP: The Breast Cancer Continuum: insights from the tamoxifen trials impact future drug development strategies. Ann N Y Acad Sci 2001, 949:327-332.

3. Sanders ME, Schuyler PA, Dupont WD, et al.: The natural history of low-grade ductal carcinoma in situ of the breast in women treated by biopsy only revealed over 30 years of long-term followup. Cancer 2005, 103:2481-2484.

4. Page DL, Dupont WD, Rogers LW, et al.: Intraductal carcinoma of the breast: follow-up after biopsy only. Cancer 1982, 49:751-758.

5. Betsill WL Jr, Rosen PP, Lieberman PH, et al.: Intraductal carcinoma. Long-term follow-up after treatment by biopsy alone. JAMA 1978, 239:1863-1867. 
6. Eusebi V, Feudale E, Foschini MP, et al.: Long-term follow-up of in situ carcinoma of the breast. Semin Diagn Pathol 1994, 11:223-235.

7. Smith GL, Smith BD, Haffty BG: Rationalization and regionalization of treatment for ductal carcinoma in situ of the breast. Int $\mathbf{J}$ Radiat Oncol Biol Phys 2006, 65:1397-1403.

8. Silverstein MJ, Cohlan BF, Gierson ED, et al.: Duct carcinoma in situ: 227 cases without microinvasion. Eur J Cancer 1992, 28:630-634.

9. Rakovitch E, Franssen E, Kim J, et al.: A comparison of risk perception and psychological morbidity in women with ductal carcinoma in situ and early invasive breast cancer. Breast Cancer Res Treat 2003, 77:285-293.

10. Partridge A: Risk perceptions and psychosocial outcomes of women with ductal carcinoma in situ: longitudinal results from a cohort study. J Natl Cancer Inst 2007, 100:243-251

11. Cooper A: On the Anatomy of the Breast. London: Longman, Orme, Green, Brown and Longmans; 1840

12. Hovey RC, Trott JF, Vonderhaar BK: Establishing a framework for the functional mammary gland: from endocrinology to morphology. J Mammary Gland Biol Neoplasia 2002, 7:17-38.

13. Teboul M: A new concept in breast investigation: echohistological acino-ductal analysis or analytic echography. Biomed Pharmacother 1988, 42:289-295.

14. Moffat DF, Going JJ: Three dimensional anatomy of complete duct systems in human breast: pathological and developmental implications. J Clin Pathol 1996, 49:48-52.

15. Love SM, Barsky SH: Anatomy of the nipple and breast ducts revisited. Cancer 2004, 101:1947-1957.

16. Cooper A: The Anatomy and Diseases of the Breast. Philadelphia: Lea and Blanchard; 1845.

17. Dietz JR, Crowe JP, Grundfest S, et al.: Directed duct excision by using mammary ductoscopy in patients with pathologic nipple discharge. Surgery 2002, 132:582-587; discussion 587-588.

18. Dooley WC, Ljung BM, Veronesi U, et al.: Ductal lavage for detection of cellular atypic in women at high risk for breast cancer. J Natl Cancer Inst 2001, 93:1624-1632.

19. Page DL, Dupont WD, Rogers LW, Rados MS: Atypical hyperplastic lesions of the female breast. A long-term follow-up study. Cancer. 1985, 55:2698-2708.

20. Wrensch M, Petrakis NL, King EB, et al.: Breast cancer risk associated with abnormal cytology in nipple aspirates of breast fluid and prior history of breast biopsy. Am J Epidemiol 1993, 137:829-833.

21. Wrensch MR, Petrakis NL, Miike R, et al.: Breast cancer risk in women with abnormal cytology in nipple aspirates of breast fluid. J Natl Cancer Inst 2001, 93:1791-1798.

22. Fabian CJ, Kimler BF, Zalles CM, et al.: Short-term breast cancer prediction by random periareolar fine-needle aspiration cytology and the Gail risk model. J Natl Cancer Inst 2000, 92:1217-1227.

23. Khan SA, Wiley EL, Rodriguez N, et al.: Ductal lavage findings in women with known breast cancer undergoing mastectomy. $\mathrm{J}$ Natl Cancer Inst 2004, 96:1510-1517.

24. Maddux AJ, Ashfaq R, Naftalis E, et al:: Patient and duct selection for nipple duct lavage. Am J Surg 2004, 188:390-394.

25. Brogi E, Robson M, Panageas KS, et al.: Ductal lavage in patients undergoing mastectomy for mammary carcinoma: a correlative study. Cancer 2003, 98:2170-2176.

26. Wood ME, Stanley MA, Crocker AM, et al.: Ductal lavage of cancerous and unaffected breasts: procedure success rate and cancer detection. Acta Cytol 2009, 53:410-415.

27. Sartorius OW, Smith HS, Morris P, et al.: Cytologic evaluation of breast fluid in the detection of breast disease. J Natl Cancer Inst 1977, 59:1073-1080.

28. Chatterton RT Jr, Geiger AS, Khan SA, et al.: Variation in estradiol, estradiol precursors, and estrogen-related products in nipple aspirate fluid from normal premenopausal women. Cancer Epidemiol Biomarkers Prev 2004, 13:928-935.
29. Khan SA, Wolfman JA, Segal L, et al.: Ductal lavage findings in women with mammographic microcalcifications undergoing biopsy. Ann Surg Oncol 2005, 12:689-696.

30. King BL, Tsai SC, Gryga ME, et al.: Detection of chromosomal instability in paired breast surgery and ductal lavage specimens by interphase fluorescence in situ hybridization. Clin Cancer Res 2003, 9:1509-1516.

31. Krishnamurthy S, Zhao L, Hayes K, et al.: Feasibility and utility of using chromosomal aneusomy to further define the cytologic categories in nipple aspirate fluid specimens: a preliminary study. Cancer 2004, 102:322-327.

32. Adduci KM, Annis CE, DeVries S, et al.: Fluorescence in situ hybridization of ductal lavage samples identifies malignant phenotypes from cytologically normal cells in women with breast cancer. Cancer 2007, 111:185-191.

33. Zhang W, Yang YC, Zhang BN, et al.: Biomarker analysis on breast ductal lavage cells in women with and without breast cancer. Int J Cancer 2006, 119:359-364.

34. Krassenstein R, Sauter E, Dulaimi E, et al.: Detection of breast cancer in nipple aspirate fluid by $\mathrm{CpG}$ island hypermethylation. Clin Cancer Res 2004, 10:28-32.

35. Fackler MJ, Malone K, Zhang Z, et al.: Quantitative multiplex methylation-specific PCR analysis doubles detection of tumor cells in breast ductal fluid. Clin Cancer Res 2006, 12:3306-3310.

36. Sauter ER, Shan S, Hewett JE, et al.: Proteomic analysis of nipple aspirate fluid using SELDI-TOF-MS. Int J Cancer 2005, 114:791-796.

37. Uchida K, Fukushima H, Toriumi Y, et al.: Mammary ductoscopy: current issues and perspectives. Breast Cancer 2009, 16:93-96.

38. •- Kapenhas-Valdes E, Feldman SM, Cohen JM, et al.: Mammary ductoscopy for evaluation of nipple discharge. Ann Surg Oncol 2008, 15:2720-2727. The authors present a single-institution study of 110 ducts in 93 patients evaluated with ductoscopy, including patient presentation, ductoscopic/pathologic findings, and surgical outcome. The potential for future diagnostic and therapeutic applications of this procedure is discussed.

39. •- Dooley WC: Ductoscopy technique - using ductoscopy anatomically direct breast conservation surgery. From 6th International Symposium on the Intraductal Approach to Breast Cancer. Santa Monica, CA. BMC Proceedings 2009, 3(Suppl 5):S16. This collection of monographs represents the current state of the art in intraductal therapy and includes brief summaries of recent and ongoing studies of intraductal therapy for both benign and malignant breast disease. Both practical and theoretical considerations for intraductal therapy are reviewed.

40. Murata S, Kominsky SL, Vali M, et al.: Ductal access for prevention and therapy of mammary tumors. Cancer Res 2006, 66:638-645.

41. Stearns V, Jacobs L, Khouri N, et al.: A phase I study assessing the feasibility and safety of intraductal pegylated liposomal doxorubicin (PLD) in women awaiting mastectomy. From 6th International Symposium on the Intraductal Approach to Breast Cancer. Santa Monica, CA. BMC Proceedings 2009, 3(Suppl 5):S28

42. Rao JY, Yang H, Zhang BN, et al.: Histopathological responses to a short term intraductal cytotoxic agent treatment: results of a feasibility study, 6th International Symposium on the Intraductal Approach to Breast Cancer. Santa Monica, CA. BMC Proceedings 2009, 3(Suppl 5):S30.

43. Love S, Zhang BN, Zhang W, et al.: Local drug delivery to the breast: a phase I study of breast cytotoxic agent administration prior to mastectomy, 6th International Symposium on the Intraductal Approach to Breast Cancer. Santa Monica, CA. BMC Proceedings, 2009, 3(Suppl 5):S29

44. •• Mahoney M, Mills D, Love S: Intraductal therapy of DCIS with liposomal doxorubicin: a preoperative trial in rural California, 6th International Symposium on the Intraductal Approach to Breast Cancer. Santa Monica, CA. BMC Proceedings 2009, 3(Suppl 5):S31. This collection of monographs represents the current state of the art 
in intraductal therapy and includes brief summaries of recent and ongoing studies of intraductal therapy for both benign and malignant breast disease. Both practical and theoretical considerations for intraductal therapy are reviewed.

45. Bhandare D, Nayar R, Bryk M, et al.: Endocrine biomarkers in ductal lavage samples from women at high risk for breast cancer. Cancer Epidemiol Biomarkers Prev 2005, 14:2620-2627.

46. •- Patil DB, Lankes HA, Nayar R, et al.: Reproducibility of ductal lavage cytology and cellularity over a 6 month interval in high risk women. Breast Cancer Res Treat 2008, 112:327-333. This interesting study explores whether ductal lavage may be used to serially follow patients at high risk for breast cancer. This may be of potential relevance in the future if both therapy and followup of treatment are to be managed through an intraductal approach.

47. Khan SA, Lankes HA, Patil DB, et al.: Ductal lavage is an inefficient method of biomarker measurement in high-risk women. Cancer Prev Res 2009, 2:265-273. 